\title{
Alterations of neurogenesis during development of Alzheimer's disease-like pathology in OXYS rats
}

\author{
A.O. Burnyasheva*, N.G. Kolosova, N.A. Stefanova, E.A. Rudnitskaya \\ Institute of Cytology and Genetics SB RAS, Novosibirsk, Russia \\ *e-mail: burn.alena2505@mail.ru
}

Key words: neurogenesis, Alzheimer's disease, OXYS rats

\begin{abstract}
Motivation and Aim: Neurogenesis is one of the major mechanisms of neuronal plasticity and it is crucial for successful learning. Neurogenesis is altered in a couple of pathological conditions including Alzheimer's disease (AD). Alteration of neurogenesis in turn results in further loss of neuronal plasticity triggering vicious circle of neurodegeneration. To investigate the link between changes of neurogenesis and development of AD we used OXYS rats which are considered as a suitable model of the most common sporadic form of AD.

Methods and Algorithms: 3- and 18-months-old male OXYS and Wistar (control) rats were used. Immunohistochemistry was used to identify the number of neuronal cells at different stages of maturation and amyloid- $\beta(\mathrm{A} \beta)$ deposition in the dentate gyrus (DG) of the hippocampus. Animal's learning and memory were evaluated in the Morris water Maze (MWM).
\end{abstract}

Results: We have shown that 3-months-old Wistar rats were able to find hidden platform in the MWM already at the $2^{\text {nd }}$ day of training while OXYS rats failed to remember the location of the platform thus demonstrating unsuccessful learning. After relocation of the platform to the opposite quadrant on the $6^{\text {th }}$ trial day Wistar rats successfully found it already at the $7^{\text {th }}$ training day while OXYS rats failed thus demonstrating alterations of reversal learning. Moreover, OXYS rats demonstrated alterations of reference memory: they spent much less time in the target quadrant at $11^{\text {th }}$ trial day, when the platform was removed from the pool. Altered learning and spatial memory may reflect changes of neuronal plasticity in the hippocampus. Indeed, we shown decreased density of neuroblasts in the DG of 3-months-old OXYS rats compared to Wistar rats. The density of neuroblasts and immature neurons dramatically decreased with age in the DG of both strains. However, the density of immature neurons was higher in the DG of 18-monthsold OXYS rats compared to Wistar rats against background of pronounced A $\beta$ deposition. Increased density of immature neurons may reflect alterations of neuronal maturation in the DG of OXYS rats.

Conclusion: Manifestation of AD-like pathology in OXYS rats occur against background of significant learning and spatial memory deteriorations as well as alterations of hippocampal neurogenesis. Progression of AD-like pathology in OXYS rats occur against background of pronounced $A \beta$ deposition and altered neuronal maturation in the DG of hippocampus.

Acknowledgements: This work was supported by grant from the Russian Foundation for Basic Research (project No. 18-015-00320). 